\title{
SOME FIXED POINT THEOREMS AND CONVOLUTION EQUATIONS
}

\author{
D. D. ANG AND D. E. DAYKIN
}

The Banach contraction principle, which is so useful in functional analysis, has been extended by various writers. One extension, due to Edelstein [2], reads as follows:

Let $F$ be a self-map of a metric space $E$ such that $\delta(F(x), F(y))$ $<\delta(x, y)$ for all $x \neq y$. Suppose there exists a point $x_{0}$ such that the sequence $x_{n}=F^{n} x_{0}$ contains a subsequence converging to a point $u$ of $E$. Then $u$ is the unique fixed point of $F$.

Edelstein's result was applied by Edwards [4] to prove the existence of solutions of certain convolution equations. We were led, by our interest in Edwards' paper, to a generalization (Theorem 1) of Edelstein's theorem. Our generalization deals with topological spaces which need not be metrizable. The value of our generalization is enhanced by the facts that it is not always obvious whether or not a space is metrizable, and even if a space is metrizable it usually is not easy to construct a metric suitable for Edelstein's theorem.

The fixed point theorems to be derived in $\$ 1$ will be applied to a study of some convolution equations in $\$ 2$.

\section{Some fixed point theorems.}

Theorem 1. Let $E$ be a topological space and let $D$ be a family of pseudometrics which are continuous on $E \times E$. Let $F$ be a self-map of $E$ such that

(i) $\delta(F x, F y) \leqq \delta(x, y)$ for all $x, y \in E$ and $\delta \in D$, and

(ii) for each $x \neq y$ there is $\delta \in D$ for which

$$
\delta(F x, F y)<\delta(x, y) .
$$

Suppose there is a point $x_{0}$ in $E$ such that the sequence of iterates $F^{n} x_{0}$ has a cluster value $u$ in $E$. Then $u$ is the unique fixed point of $F$. Furthermore $\delta\left(F^{n} x_{0}, u\right) \rightarrow 0$ for each $\delta \in D$.

Proof. Suppose $u^{\prime} \neq u^{\prime \prime}$ are two fixed points of $F$. Then there is a $\delta \in D$ such that $\delta\left(u^{\prime}, u^{\prime \prime}\right)=\delta\left(F u^{\prime}, F u^{\prime \prime}\right)<\delta\left(u^{\prime}, u^{\prime \prime}\right)$, which is impossible. Hence $F$ can have at most one fixed point, and we will show that $u$ is a fixed point of $F$.

Put $v=F u$, and suppose that $u \neq v$. Then by (ii) we have

Received by the editors March 1, 1967. 


$$
\delta(F u, F v)<\delta(u, v)
$$

for some $\delta \in D$. From now on we only deal with this particular one $\delta$ of the family $D$ of pseudometrics. We denote by $E_{\delta}$ the pseudometric space defined on $E$ by $\delta$. In $E_{\delta} \times E_{\delta}$, the map $(x, y) \rightarrow(F x, F y)$ is continuous, and hence the map $(x, y) \rightarrow \delta(F x, F y) / \delta(x, y)$ is continuous at $(u, v)$. Thus it follows from (1) that there is a $k<1$ and a neighbourhood $U \times V$ of $(u, v)$ in $E_{\delta} \times E_{\delta}$ such that

$$
\delta(F x, F y)<k \delta(x, y) \quad \text { for all }(x, y) \in U \times V .
$$

Now there exists a subsequence $x_{r}=F^{n} x^{n} x_{0}$ of $F^{n} x_{0}$ which converges to $u$ in $E_{\delta}$, i.e. $\delta\left(x_{r}, u\right) \rightarrow 0$, and from (i) we also have $\delta\left(F x_{r}, F u\right) \rightarrow 0$, and we recall that $F u=v$. Hence there exists an integer $N$ such that

$$
x_{r} \in U \text { and } F x_{r} \in V \quad \text { for } r \geqq N \text {, }
$$

and so

$$
\delta\left(F x_{r}, F F x_{r}\right)<k \delta\left(x_{r}, F x_{r}\right) \quad \text { for } r \geqq N .
$$

By repeated application of (i), we have

$$
\delta\left(F^{m} x_{r}, F^{m} F x_{r}\right) \leqq \delta\left(F x_{r}, F F x_{r}\right),
$$

for $m=1,2,3, \cdots$, and in particular, putting $m=n_{r+1}-n_{r}$,

$$
\delta\left(x_{r+1}, F x_{r+1}\right) \leqq \delta\left(F x_{r}, F F x_{r}\right) .
$$

Using (2), (3) and the triangle inequality gives us

$$
\delta\left(x_{r+1}, F x_{r+1}\right)<k \delta\left(x_{r}, F x_{r}\right) \leqq k \delta\left(x_{r}, u\right)+k \delta(u, v)+k \delta\left(v, F x_{r}\right) .
$$

Taking the limit $r \rightarrow \infty$ yields

$$
\delta(u, v) \leqq k \delta(u, u)+k \delta(u, v)+k \delta(v, v)=k \delta(u, v),
$$

and it follows from this contradiction that $u=v$. Hence $u$ is a fixed point of $F$. Let $\delta \in D$ and let $\epsilon_{0}>0$. Since $u$ is a cluster value of $\left(F^{n} x_{0}\right)$, there exists an $n_{0}$ such that $\delta\left(F^{n} x_{0}, u\right)<\epsilon_{0}$. Now for each $n \geqq n_{0}$,

$$
\delta\left(F^{n} x_{0}, u\right)=\delta\left(F^{n} x_{0}, F^{n} u\right) \leqq \delta\left(F^{n_{0}} x_{0}, F^{n_{0}} u\right)<\epsilon_{0} .
$$

Hence $\delta\left(F^{n} x_{0}, u\right) \rightarrow 0$ and the theorem is proved.

CoROLlary. Let $s \geqq 1$ be a fixed integer. If the conditions of Theorem 1 hold with $F$ replaced by $F^{s}$, then $u$ is the unique fixed point of $F$.

Proof. We know by Theorem 1 that $u$ is the unique fixed point of $F^{s}$. Since $F u$ is also a fixed point of $F^{s}$, by uniqueness we have $F u=u$. 
Theorem 2. Let $M$ be a subset of a topological vector space E. Let $P$ be a family of continuous seminorms on $E$. Let $F$ be a self-map of $M$ such that

(i) $p(F x-F y) \leqq p(x-y)$ for all $p \in P$, and

(ii) for each $x \neq y$, there exists a $p \in P$ such that

$$
p(F x-F y)<p(x-y) .
$$

Suppose that there exists a point $x_{0}$ in $M$ such that the sequence of iterates $F^{n} x_{0}$ has a cluster value $u$ in $M$. Then $u$ is the unique fixed point of $F$. Furthermore, $p\left(F^{n} x_{0}, u\right) \rightarrow 0$ for each $p \in P$.

Proof. The theorem is an immediate consequence of Theorem 1, because the functions $\delta$ defined by $\delta(x, y)=p(x-y)$ are continuous pseudometrics.

Theorem 3. Let $E$ be a topological vector space with topological dual $E^{\prime}$. Let $A$ be an endomorphism of $E$, and $A^{\prime}$ the adjoint of $A$ which is assumed to take $E^{\prime}$ into $E^{\prime}$. Suppose that $A^{\prime}$ has a family $G$ of eigenvectors each of which belongs to an eigenvalue of modulus less than 1. Assume further that $G$ is total over $E$. Let $y_{0} \in E$ be given. Suppose there is a point $x_{0}$ in $E$ such that the sequence of iterates

$$
x_{n}=A^{n} x_{0}+y_{0}+A y_{0}+\cdots+A^{n-1} y_{0}
$$

has a cluster value $u$ in $E$. Then $u$ is the unique solution of the equation $x=A x+y_{0}$.

Proof. We define a self-map $F$ of $E$ by $F x=A x+y_{0}$. Then for each $e \in G$ we define a seminorm $p$ by $p(y)=|(y, e)|$. The continuity of $p$ follows from the fact that $G \subset E^{\prime}$. We have the inequality

$$
\begin{aligned}
p(F x-F y) & =|(F x-F y, e)|=|(A x-A y, e)|=\left|\left(x-y, A^{\prime} e\right)\right| \\
& =|(x-y, \lambda e)| \leqq|(x-y, e)|=p(x-y),
\end{aligned}
$$

where $\lambda$ is the eigenvalue corresponding to the eigenvector $e$, and by hypothesis $|\lambda|<1$. Furthermore since $G$ is total over $E$, for each $x \neq y$ there is an $e \in G$ such that $(x-y, e) \neq 0$, and hence for the corresponding $p$ the above inequality is strict. Thus we have shown that all the conditions of Theorem 2 are satisfied, and the result follows.

The endomorphism $A$ of Theorem 3 need not be continuous, but the conditions of the theorem imply that it is weakly continuous. Since $G$ is total over $E$, the set $E^{\prime}$ separates points of $E$, so $\left(E, E^{\prime}\right)$ is a dual pair. We also have $A^{\prime}\left(E^{\prime}\right) \subseteq E^{\prime}$ and hence $A$ is weakly continuous, by Proposition 12 [8, p. 38]. 
REMARKs. In [5] Fearnley-Sander proved the particular case of Theorem 1 with the stronger condition that $F^{n} x_{0}$ contains a convergent subsequence.

Monna has a result [7, Theorem 1] where $E$ (in our terminology) is a complete Hausdorff uniform space described by a family of pseudometrics such that

$$
\delta(F x, F y) \leqq k_{\delta} \delta(x, y), \quad 0<k_{\delta}<1,
$$

for each $x, y$ such that $\delta(x, y) \leqq A=A(\delta)$. Another interesting result is by Edelstein [3, Theorem 4] where $E$ (in our terminology) is a uniform space which is $U$-chained (cf. [10, p. 204]). These results are related but mutually independent. We would like to thank the referee for drawing them to our attention.

Our Theorem 2 is a generalization of Edwards' main theorems, (Theorems 1 and 2 of [4]), if we exclude the case of non-Hausdorff spaces which have little applications in the present context. To see this, let $E_{1}$ be a vector space and $P$ be a countable family of seminorms on $E_{1}$. Let $\left(E_{1}, P\right)$ be the set $E_{1}$ endowed with the weakest topology on $E_{1}$ such that the seminorms in $P$ be continuous. Then our theorem implies a result stronger than Edwards' Theorems 1 and 2 by taking $\left(E_{1}, P\right)$ for our $E$. (The fact that $p\left(F^{n} x_{0}, u\right) \rightarrow 0$ for each $p \in P$ is of course equivalent to the convergence of $\left(F^{n} x_{0}\right)$ to $u$ in the weak topology defined by the seminorms in $P$.) In [5], FearnleySander proved a result lying between Edwards' Theorem 2 and our Theorem 2. Our Theorem 3 is a generalization of Edwards' Theorem 3 , since our family of eigenvectors need not be countable or strongly bounded; nor does our sequence of iterates need to contain a bounded subsequence converging to a cluster value of the sequence.

In the applications of Theorem 2 to be given in $\$ 2$ the existence of a cluster value of the sequence $\left(F^{n} x_{0}\right)$ of iterates is due to the (in some sense) weak compactness of certain subsets of a certain Banach space. However, unless the space is first countable, compactness and sequential compactness are in general not equivalent.

2. Some convolution equations. We commence with some preliminary considerations. In what follows, $G$ will be a compact Hausdorff group, $C(G)$ the space of continuous functions on $G$, and $M(G)$ its topological dual. The elements of $M(G)$ are the Radon measures on $G$. By $\sigma(M(G))$ we shall mean the set $M(G)$ with the weak*togology.

Let $\left(U_{i}\right), i \in I$, be a complete set of inequivalent, irreducible (finite-dimensional) unitary continuous representations of $G$ (such a 
set exists by a theorem of Gelfand-Raikov [6]). As in [4], we set for each $U_{i}$

$$
\hat{x}\left(U_{i}\right)=\int_{G} U_{i}(t) d x(t), \quad x \in M(G) .
$$

Then each $\hat{x}\left(U_{i}\right)$ is an endomorphism of the representation space of $U_{i}$. Let its norm be denoted by $\left\|\hat{x}\left(U_{i}\right)\right\| \equiv p_{i}(x)$. Then each $p_{i}$ is a continuous seminorm on $\sigma(M(G))$. Furthermore, since the coordinates of the representations $U_{i}$ are dense in $C(G)$, it follows that for each $x \neq 0$ in $M(G)$, there exists a $p_{i}$ such that $p_{i}(x) \neq 0$. Let $\mu \in M(G)$ and $x \in M(G)$ (possibly $x \in L^{p}(G)$ ), then $\mu * x$ will be the convolution product of $\mu$ and $x$. It can be shown that

$$
p_{i}(\mu * x) \leqq p_{i}(\mu) p_{i}(x) .
$$

We shall be concerned with the existence of solutions of convolution equations of the form

$$
x-\mu * x=y,
$$

where $\mu \in M(G)$ and $y$ is a given element of $M(G)$ (possibly $L^{p}(G)$ ). The existence of solutions of $\left(^{*}\right)$ in $L^{p}, 1<p<\infty$ and in $M(G)$ was considered by Edwards [4]. We shall apply the fixed point theorems of the preceding section to solve $\left(^{*}\right)$ in $M(G)$, and also in $L^{1}(G)$.

We first remark that $x$ is a solution of $\left({ }^{*}\right)$ iff $x$ is a fixed point of the operator $F$ defined by

$$
F x=\mu * x+y .
$$

Thus in what follows, theorems will be couched in the language of fixed point theorems. As usual, we set

$$
\begin{aligned}
& F^{n} x=F F^{n-1} F x, \quad n=1,2, \cdots, \\
& F^{0} x=x .
\end{aligned}
$$

Theorem 4. Let $\mu \in M(G)$ be such that $p_{i}(\mu)<1, i \in I$. Let $y \in M(G)$. Then either

(a) $\lim _{n \rightarrow \infty}\left\|F^{n} x\right\|=+\infty$ for each $x \in M(G),\|\cdot\|$ being the usual norm in $M(G)$, or

(b) $F$ has a unique fixed point in $M(G)$.

Proof. Suppose (a) is false and let $x_{0}$ be an element of $M(G)$ such that $\left(F^{n} x_{0}\right)$ contains a bounded subsequence $\left(F^{n_{k}} x_{0}\right)$. Since $\left(F^{n_{k}} x_{0}\right)$ is conditionally compact in $\sigma(M(G))$, it has a cluster value $u$ in $\sigma(M(G))$. A fortiori, $u$ is a cluster value of $\left(F^{n} x_{0}\right)$ in $\sigma(M(G))$. Now from the inequality (4) and since $p_{i}(\mu)<1, i \in I$, we have 


$$
p_{i}(F x-F y) \leqq p_{i}(x-y), \quad i \in I,
$$

the inequality being strict if $p_{i}(x-y) \neq 0$. Theorem 2 can now be applied with $E=\sigma(M(G))$ to conclude that $u$ is the unique fixed point of $F$.

REMARKs. The preceding theorem was proved by Edwards with some countability conditions imposed on the family $\left(U_{i}\right)$.

Equation (*) was solved in $L^{p}(G), 1<p<\infty$, by Edwards (cf. $[4$, Theorem 4$])$. The relative facility with which we could deal with $M(G)$ and also with $L^{p}(G),(1<p<\infty)$ is due to the existence of simple compactness criteria for these spaces in the weak* (and weak) sense, respectively. The case of $L^{1}(G)$ is the most difficult, owing to the fact that the corresponding compactness criteria are difficult to handle. The situation is not so hopeless in the case where $G$ is Abelian, since we can (and shall) use the known techniques of Fourier transforms.

In what follows, $G$ will be a compact Hausdorff Abelian group, $\Gamma$ its character group, its elements being denoted by $\gamma$. For $x \in L^{1}(G)$, $\mu \in M(G)$, we shall denote by $\hat{x}(\gamma), \hat{\mu}(\gamma)$ their Fourier transform and Fourier-Stieltjes transform, respectively. The set of Fourier transforms will be denoted by $A(\Gamma)$, the set of Fourier-Stieltjes transforms will be denoted by $B(\Gamma)$.

Theorem 5. Suppose $G$ Abelian. Let $y \in L^{1}(G)$ and $\mu \in M(G)$ be such that

(i) the sequence $\lambda_{n} \equiv \sum_{i+0}^{n} \mu^{i}$ contains a bounded subsequence, the $\mu^{i}$ being convolution powers and $\mu^{0}$ being the unit of $M(G)$ and

(ii) $|\hat{\mu}(\gamma)|<1, \quad \gamma \in \Gamma$.

Then $F$ has a unique fixed point in $L^{1}(G)$.

Proof. Let $\left(\lambda_{n_{k}}\right)$ be a bounded subsequence of $\left(\lambda_{n}\right)$. We have by direct computation

$$
F^{n} y=y+\mu * y+\cdots+\mu^{n} * y .
$$

The sequence $\left(F^{n} k y\right)$ is clearly bounded in $M(G)$ and hence has a cluster value $\lambda$ in $\sigma(M(G))$. Taking Fourier transforms of both sides of (6) gives

$$
\left(F^{n} y\right)^{\wedge}(\gamma)=\hat{y}(\gamma)+\hat{\mu}(\gamma) \hat{y}(\gamma)+\cdots+\hat{\mu}^{n}(\gamma) \hat{y}(\gamma) .
$$

This sequence converges (in view of (ii)) to $\hat{y}(\gamma)(1-\hat{\mu}(\gamma))^{-1}$. Hence its subsequence $\left(F^{n k} y\right)^{\wedge}(\gamma)$ also converges to the same limit for each $\gamma$. But convergence of $\left(F^{n k} y\right)^{\wedge}(\gamma)$ for each $\gamma$ implies that $\left(F^{n k} y\right)$ is a Cauchy sequence in $\sigma(M(G))$, since the characters are fundamental in 
$C(G)$. Hence $\left(F^{n_{k} y}\right)$ converges to its cluster value $\lambda$ in the topology of $\sigma(M(G))$. This implies

$$
\hat{\lambda}(\gamma)=\hat{y}(\gamma)(1-\hat{\mu}(\gamma))^{-1} .
$$

We next show that $\hat{y}(\gamma)(1-\hat{\mu}(\gamma))^{-1} \in A(\Gamma)$. Now $(1-\hat{\mu}(\gamma))^{-1}$ is continuous since $\Gamma$ is discrete. Furthermore,

$$
(1-\hat{\mu}(\gamma))^{-1}=\lim _{n} \hat{\lambda}_{n}(\gamma)=\lim _{k} \hat{\lambda}_{n_{k}}(\gamma) .
$$

By setting

$$
\left\|\lambda_{n_{k}}\right\| \equiv\left\|\lambda_{n_{k}}\right\| \quad\left(=\text { norm of } \lambda_{n_{k}} \text { in } M(G)\right),
$$

we have by (i)

$$
\left\|\hat{\lambda}_{n_{k}}\right\| \leqq M_{0} \quad \text { for a certain constant } M_{0} .
$$

Hence it follows from a theorem of Eberlein ([1]; cf. also [9, p. 34]) that $(1-\hat{\mu}(\gamma))^{-1} \in B(\Gamma)$. Hence $\lambda(\gamma) \in A(\Gamma)$, in other words, there exists a $u \in L^{1}(G)$ such that $d \lambda=u d t$, where $d t$ is the Haar measure on $G$. We thus have proved the existence of a $u \in L^{1}(G)$, which is a cluster value of $\left(F^{n} y\right)$ in $\sigma\left(L^{1}\right)$, where $\sigma\left(L^{1}\right)$ is the set $L^{1}(G)$ with the topology induced by $\sigma(M(G))$. Let us set, as previously,

$$
p_{\gamma}(x)=|\hat{x}(\gamma)|, \quad p_{\gamma}(\mu)=|\hat{\mu}(\gamma)| .
$$

Then $\left(p_{\gamma}\right), \gamma \in \Gamma$, is a family of seminorms continuous on $\sigma\left(L^{1}\right)$ and such that $p_{\gamma}(F x-F y) \leqq p_{\gamma}(x-y)$, the inequality being strict if $p_{\gamma}(x-y) \neq 0$. Furthermore, for each $x \neq 0$ in $L^{1}(G)$, there exists a character $\gamma$ such that $p_{\gamma}(x) \neq 0$. The conditions of Theorem 2 are hence satisfied with $E=\sigma\left(L^{1}\right)$. Thus $u$ is the unique fixed point of $F$.

Remarks. The condition (i) of Theorem 5 is stronger than the following condition:

(iii) For a certain $x_{0} \in L^{1}(G)$, the sequence $\left(F^{n} x_{0}\right)$ contains a bounded subsequence.

It can be shown that if $y$ is a positive definite element of $L^{1}(G)$ and $1-\hat{\mu}$ is bounded away from zero, then the conclusion of Theorem 5 remains valid with condition (i) replaced by the weaker condition (iii). The proof proceeds on similar lines as for Theorem 5, noting that $|1-\hat{\mu}(\gamma)|^{-1}$ is bounded. Since $\hat{y}(\gamma)$ is integrable on $\Gamma$, it follows that $\hat{y}(\gamma)(1-\hat{\mu}(\gamma))^{-1}$ is integrable. By an application of Eberlein's theorem (loc. cit.), we also have $\hat{y}(\gamma)(1-\hat{\mu}(\gamma))^{-1} \in B(\Gamma)$. Hence $\lambda$ defined in the proof of Theorem 5 is absolutely continuous and the result follows.

Note ADDED IN PROOF. For the proof of Theorem 5, it would have been sufficient to prove that $(1-\hat{\mu})^{-1}$ is in $B(\Gamma)$ and then apply the 
Fourier inversion formula. However the given proof would apply with minor modifications to the following stronger result.

THEOREM 6. Let $G$ be a locally compact abelian group. Suppose

(a) that $|\hat{\mu}(\gamma)|<1$ for $\gamma$ in a subset $\Gamma_{0}$ of $\Gamma$ such that for each nonzero $x$ in $L^{1}(G)$ there exists a $\gamma$ in $\Gamma_{0}$ for which $\hat{x}(\gamma) \neq 0$, and

(b) that $\left(\sum_{0}^{n} \hat{\mu}^{i}(\gamma)\right)$ converges to a continuous function on $\Gamma$. Suppose furthermore that the condition (i) of Theorem 5 holds. Then $F$ has a unique fixed point in $L^{1}(G)$ for each $y$ in $L^{1}(G)$.

(If $G$ is compact, then $\Gamma_{0}=\Gamma$, and (b) follows from (a).)

\section{REFERENCES}

1. IV. F. Eberlein, Characterization of Fourier-Stieltjes transforms, Duke Math. J. 22 (1955), 465-468.

2. M. Edelstein, On fixed and periodic points under contractive mappings, J. London Math. Soc. 37 (1962), 74-79.

3. - On non-expansive mappings of uniform spaces, Indag. Math. 23 (1961), 89-96.

4. R. E. Edwards, A fixed point theorem with applications to convolution equations, J. Austral. Math. Soc. 3 (1963), 385-395.

5. D. Fearnley-Sander, Contraction mappings in uniform spaces, 1963, (unpublished).

6. E. Hewitt and K. A. Ross, Abstract harmonic analysis, Academic Press, New York, 1963.

7. F. Monna, Sur un theoreme de M. Luxemburg concernaut les points fixes d'une classe d'applications d'un espace metrique dans lui même, Indag. Math. 27 (1965), 4751.

8. A. P. Robertson and W. J. Robertson, Topological vector spaces, Cambridge Univ. Press, Cambridge, 1964.

9. W. Rudin, Fourier analysis on groups, Interscience, New York, 1962.

10. N. Bourbaki, General topology, Part 1, Addison-Wesley, Reading, Mass., 1966 ; p. 204.

UNIVERSITY OF SAIGON AND

UNIVERSITY OF MALAYA 